\title{
Operando monitoring the insulator-metal transition of $\mathrm{LiCoO}_{2}$
}

\author{
Eibar Flores $^{\mathrm{a} *}$, Nataliia Mozhzhukhina ${ }^{\mathrm{b}}$, Ulrich Aschauer ${ }^{\mathrm{c}}$, Erik J. Berg ${ }^{\mathrm{b}}$ \\ a: Electrochemistry Laboratory, Paul Scherrer Institute, 5232 Villigen, Switzerland \\ b: Department of Chemistry, Ångström Laboratory, Uppsala University, Box 538, SE-751 21 Uppsala, Sweden. \\ c: Department of Chemistry and Biochemistry, University of Bern, CH-3012 Bern, Switzerland.
}

\begin{abstract}
LiCoO}_{2}$ (LCO) is one of the most-widely used cathode active materials for Li-ion batteries. Even though the material undergoes an electronic two-phase transition upon Li-ion cell charging, LCO exhibits competitive performance in terms of rate capability. Herein the insulator-metal transition of LCO is investigated by operando Raman spectroscopy complemented with DFT calculations and a newly-developed sampling volume model. We confirm the presence of a Mott insulator $\alpha$-phase at dilute Li-vacancy concentrations ( $\mathrm{x}>$ 0.87 ) that transforms into a metallic $\beta$-phase at $x<0.75$. In addition, we find that the charge-discharge intensity trends of LCO Ramanactive bands exhibit a characteristic hystheresis, which, unexpectedly, narrows at higher cycling rates. When comparing these trends to a newly-developed numerical model of laser penetration into a spatially-heterogeneous particle we provide compelling evidence that the insulator-metal transition of $\mathrm{LiCoO}_{2}$ follows a two-phase route at very low cycling rates, which is suppressed in favor of a solid-solution route at rates above $10 \mathrm{~mA} / \mathrm{g}_{\mathrm{LCO}}(\sim \mathrm{C} / 10)$. The observations explain why $\mathrm{LiCoO}_{2}$ exhibits competitive rate capabilities despite being observed to undergo an intuitively slow two-phase transition route: a kinetically faster solid-solution transition route becomes available when the active material is cycled at rates $>\mathrm{C} / 10$. Operando Raman spectroscopy combined with sample volume modelling and DFT calculations is shown to provide unique insights into fundamental processes governing the performace of state-of-the-art cathode materials for $\mathrm{Li}$-ion batteries.
\end{abstract}

\section{Introduction}

$\mathrm{LiCoO}_{2}$, lithium cobalt oxide (LCO), is the most widely used cathode active material (CAM) for batteries in portable applications, and as such, it has been subject of intense study. LCO-based composite electrodes cycle reversibly if restricted to operate between 3.0 and $4.2 \mathrm{~V} v s$. $\mathrm{Li}^{+} / \mathrm{Li}$. Within such limits LCO delivers specific capacity of $\sim 150 \mathrm{mAh} / \mathrm{g}$ (around $0.5 \mathrm{Li}^{+}$ per formula unit). Cycling positive to $4.2 \mathrm{~V}$ triggers multiple undesired processes that, individually or collectively, result in the significant loss of specific charge and rapid cell death. ${ }^{1-3}$ Accordingly, major research effors are still dedicated to stabilizing LCO's operation beyond the $4.2 \mathrm{~V}$ cutoff by, for instance, surface coatings ${ }^{4}$ and cation doping., 5

The reversible processes occuring below $4.2 \mathrm{~V}$ do however deserve more attention as a deeper understanding thereof could provide keys to further improve the performance metrics of LCO. ${ }^{7,8}$ Interestingly, LCO undergoes an insulator-metal firstorder phase transition in every cycle with no apparent adverse effect on neither its rate capability nor stability. ${ }^{9}$ The transition manifests as a flat plateau dominating the constant current profiles of the $\mathrm{LiCoO}_{2}$ composite electrode (Figure S1), which appears as a well-defined redox couple around $3.90 \mathrm{~V}$ in the differential charge plot. $^{10}$ X-ray diffraction studies had confirmed the coexistence of two hexagonal phases in the $0.93>$ $x>0.75$ SOL region ( $\mathrm{x}$ in $\mathrm{Li}_{\mathrm{x}} \mathrm{CoO}_{2}$ ), ${ }^{9}$ where (de)lithiation proceeds as a first-order phase transition involving the competing depletion and growth of two phases $-\mathrm{a} \mathrm{Li}_{0.93} \mathrm{CoO}_{2} \alpha$ phase and $\mathrm{Li}_{0.75} \mathrm{CoO}_{2} \beta$-phase - sharing the same $R \overline{3} \mathrm{~m}$ lattice symmetry with slightly different lattice parameters.

Typically, first-order phase transitions do imply the rate-limiting formation and propagation of phase boundaries, lattice mismatch and volume changes, all of which would intuitively lead to slow (de)lithiation kinetics and severe mechanical degradation of the active material. ${ }^{11}$ While X-ray diffraction is typically used to study structural phase transitions, the morphological and electronic aspects of the LCO phase transition can be better understood via spatially-resolved techniques sensitive to both crystallographic and electronic structure. We have recently demonstrated the ability of Raman spectroscopy to detect operando multiple electrode phenomena - e.g. cation ordering, structural degradation and oxygen oxidation- within single particles of Ni-rich cahode materials. ${ }^{12,13}$ Moreover, the electronic structure of LCO is succeptible to resonance Raman effects that have been observed, ${ }^{14}$ but not explored for characterization pruposes.

In this work, we exploit the spatial resolution and electronic sensitivity of Raman spectroscopy to gain new insigths into the insulator-metal phase transition of LCO. Our time-resolved methodology, coupled with the $\mu \mathrm{m}$ resolution of the laser probe, enables monitoring the Raman spectra of a single CAM particle in operation. Combining experimental data with DFT calculations highlights the changes in the electronic structure and the dynamics of the phase transition of LCO.

\section{Materials and methods}

Electrode Preparation. The composite electrodes were prepared from mixed slurries of $89 \mathrm{wt} \% \mathrm{LiCoO}_{2}$ powder (stored in an Ar-filled glovebox, Alfa Aesar, Germany), 5 wt \% polyvinylidene difluoride (PVdF Kynar HSV 9000, Arkema), $4.6 \mathrm{wt} \%$ amorphous carbon Super C65, and $1.4 \mathrm{wt} \%$ graphite SFG6 (Imerys Graphite and Carbons) dispersed in n-methyl pyrrolidone solvent (NMP, Sigma-Aldrich). The slurries were coated onto Celgard 2400 (Celgard LLC) sheets by doctor blading at a $100-\mu \mathrm{m}$ wet thickness. The coated sheets were dried for $10 \mathrm{~h}$ under dynamic vacuum at $80{ }^{\circ} \mathrm{C}$, punched to $14-\mathrm{mm}$ diameter electrodes, further dried under dynamic vacuum at 80 ${ }^{\circ} \mathrm{C}$ overnight, and finally introduced into an argon filled glovebox $\left(\mathrm{O}_{2}, \mathrm{H}_{2} \mathrm{O}<1 \mathrm{ppm}\right)$.

Raman Spectro-Electrochemical Cell Assembly. The operando Raman measurements were performed using a custommade Raman spectro-electrochemical cell, as previously 
described ${ }^{15}$. In summary, the cell was assembled in a coin-cell configuration inside an argon-filled glovebox $\left(\mathrm{O}_{2}, \mathrm{H}_{2} \mathrm{O}<1 \mathrm{ppm}\right)$. Before assembly, the composite electrodes and the Celgard 2400 separator $(\varnothing 17 \mathrm{~mm})$ were wetted for several minutes in LC30 electrolyte $\left(1.0 \mathrm{M} \mathrm{LiClO}_{4}\right.$ in $1: 1(\mathrm{w} / \mathrm{w})$ ethylene carbonate (EC)/dimethyl carbonate (DMC)). The LCO working electrodes were pressed against an aluminum mesh (Ø17 mm, 5 Al 7-125, Dexmet corporation, CT, US) for electric contact. Lithium metal disks (0.2 mm thick, Ø12 mm, Sigma-Aldrich, Germany) were used as counter electrode.

Operando Raman Measurements. The Raman spectra under red laser excitation were acquired using a Labram HR800 Raman microscope (Horiba-Jobin Yvon) equipped with a He-Ne laser $(632.8 \mathrm{~nm})$. A grating was used as dispersion element with a groove density of 600 grooves $/ \mathrm{mm}$ that attains a $2 \mathrm{~cm}^{-1}$ spectral resolution. The hole and slit of the confocal system were fixed at 1000 and $100 \mu \mathrm{m}$, respectively. The laser was focused on the sample using a $50 \times$ (numerical aperture 0.55 ) objective, which produced a laser spot of ca. $1.4 \mu \mathrm{m}$ diameter with an estimated depth of focus of around $4.2 \mu \mathrm{m} .{ }^{16}$ However, opaque samples such as $\mathrm{Li}_{x} \mathrm{MO}_{2}$ oxides strongly dampen the laser intensity profile in accordance with the dielectric properties of the sample, and the effective sampling depth might be significantly shallower than the confocal depth of focus. ${ }^{17}$ The nominal laser power was filtered down to $0.6 \mathrm{~mW} / \mathrm{um}^{2}$ to avoid sample overheating. The cell was cycled galvanostatically with a computer-controlled galvanostat (CCCC Hardware, Astrol Electronic, Switzerland) at an applied current of 10, 20 or 40 $\mathrm{mA} / \mathrm{g}_{\mathrm{LCO}}$ normalized to the weight of the LCO active material in the electrode. The Raman spectra under green laser excitation were acquired using a Renishaw InVia Raman microscope equipped with a Lieca LM optical microscope, a CCD camera and a $532 \mathrm{~nm}$ laser. A grating of 1800 grooves $/ \mathrm{mm}$ was used. The laser was focused using a long focus $50 \times$ (numerical aperture 0.5 ) objective with an expected depth of focus of c.a. $4.2 \mu \mathrm{m}$, and dampened with neutral filters to attain $0.5 \mathrm{~mW} / \mathrm{um}^{2}$ power at the sample. A portable Biologic SP-240 potentiostat was used for galvanostatic cycling of the cell under green excitation laser. In all measurements, the cell was cycled at room temperature and the Raman-probed sample spot was monitored before and after the experiment to verify that it remained in focus. Every recorded spectrum resulted from the average of minimum 5 acquisitions exposed for $100 \mathrm{~s}$ each.

Operando spectra analysis. The data treatment was performed using a custom-made Python-based program consisting of a graphical user interface (GUI) and a spectra processing engine. First, the optimum parameters for baseline correction (e.g. polynomial degree) and peak fitting (e.g. number of peaks) were found by applying it to several spectra and visually inspecting the outocmes using the GUI. The baselines were fitted parametrically using low-degree polynomials while the Raman bands were fitted using Lorentz-type profiles. Once the parameters were verified to satisfactorly fit multiple spectra samples, they were automatically applied to all hundreds of spectra from the operando experiment by the Python-based engine. The state of lithiation (SOL) was calculated from the cycling data as the fraction of the specific charge at a given time relative to the theoretical specific charge of LCO:

$$
x\left(\text { in } \mathrm{Li}_{\mathrm{x}} \mathrm{CoO}_{2}\right)=\frac{I \cdot t}{m_{\text {active }} \cdot Q_{\text {theor }}}
$$

where $I(\mathrm{~mA})$ is the galvanostatic current, $t(\mathrm{~h})$ is the elapsed cycling time, $m_{\text {active }}(\mathrm{g})$ is the mass of active material in the composite electrode and $\mathrm{Q}_{\text {theor }}$ the theoretical specific charge of $\mathrm{LiCoO}_{2}$ (i.e. $273.9 \mathrm{mAh} / \mathrm{g}$ ).

DFT calculations. The density functional theory (DFT) calculations were carried out using the VASP $\operatorname{code}^{18-20}$, using the Perdew-Burke-Ernzerhof exchange correlation functional. ${ }^{21}$ Electron-core interactions were described by PAW potentials ${ }^{22,23}$ with $\mathrm{Li}(1 \mathrm{~s}, 2 \mathrm{~s}), \mathrm{Co}(4 \mathrm{~s}, 3 \mathrm{p}, 3 \mathrm{~d})$, and $\mathrm{O}(2 \mathrm{~s}, 2 \mathrm{p})$ valence states and wavefunctions were expanded in plane waves up to a cutoff energy of $500 \mathrm{eV}$. We applied a Hubbard $U^{24,25}$ to the transition metal $d$ states with $U_{\mathrm{Co}}=4.91 \mathrm{eV} .^{26}$ The reciprocal space of the 8 -atom cell of LCO was sampled using $\Gamma$-centered meshes with dimensions $6 \times 6 \times 6$ for the rombohedral structure. All internal and cell degrees of freedom were relaxed until forces converged below $10^{-3} \mathrm{eV} / \AA$ and stresses below $5 \cdot 10^{-3} \mathrm{eV} / \AA^{3}$. Phonon frequencies were computed using the frozen phonon method as implemented in the phonopy package. ${ }^{27}$ The mode intensities were computed from the mode-dependent change in dielectric constant ${ }^{28}$ evaluated via finite differences for mode amplitudes of $\pm 0.01 \AA$ and dielectric constants computed using density functional perturbation theory. Single vacancies were created in $2 \times 1 \times 1,2 \times 2 \times 1,3 \times 3 \times 1$ and $4 \times 4 \times 1$ supercells, corresponding to $x=0.75, x=0.875, x=0.944$ and $x=0.969$. For these defective cells, only internal coordinates were relaxed and the reciprocal space meshes were adjusted according to the supercell dimensions.

\section{Results and discussion}

\section{Nature of the phase transition}

According to NMR and electric measurements, the Li-rich $\mathrm{Li}_{0.93} \mathrm{CoO}_{2}$ phase features localized electron holes and behaves as an insulator, while delocalized holes provide metallic-like conduction properties to the $\mathrm{Li}$-poor $\mathrm{Li}_{0.75} \mathrm{CoO}_{2}$ phase. ${ }^{29,30} \mathrm{DFT}$ calculations based on the local-density approximation (LDA) suggest that electron holes resulting from oxidising LCO localize due to interactions with a dilute concentration of lithium vacancies, and that this interaction is strong enough to drive phase separation. ${ }^{31}$

Our DFT generalized gradient approximation (GGA) results Figure 1a) agree with these previous findings, showing for $0.97<$ $\mathrm{x}<0.87$ a spin-polarized impurity band of Li-vacancy bound holes above the valence band edge. This is indicative of hole localization in the spin-polarized environment close to a specific Co site. The impurity band disappears at $\mathrm{x}=0.75$, where the material becomes metallic and returns to its antiferromagnetic state. It was argued that since this band is half-filled, the material should undergo a Mott transition when correlation between the Co $3 d$ electrons is strong enough. ${ }^{31}$ Our GGA $+U$ calculations accounting for static correlation (but still neglecting the dynamic correlation modelled in methods such as dynamical mean field theory - DMFT) show a signature of this transformation, where two bands become visible and more separated from the valence band upon delithiation. The band separation is especially clear at $\mathrm{x}=0.87$ (Figure $1 \mathrm{~b}$ ). We note however that at $\mathrm{x}=0.97$, i.e. in the nearly fully lithiated state, our GGA $+U$ calculations show only a single peak (Figure 1b), possibly due to the highly dilute hole and $\mathrm{Li}$ vacancy concentration. Our results thus show that $\mathrm{Li}_{\mathrm{x}} \mathrm{CoO}_{2}$ is a Mott insulator for large $\mathrm{x}$ before becoming metallic between $\mathrm{x}=0.87$ and $\mathrm{x}=0.75$.

The non-rigid behavior of the LCO electronic structure is likely to manifest in its Raman spectrum, since the changes in electron density originating from hole localization influence both the bond strengths and polarizabilites defining Raman band frequencies and intensities, respectively.

\section{Raman spectra of fully lithiated $\mathrm{LiCoO}_{2}$}

According to symmetry-based considerations (Fig. S2), fully lithiated LCO expectedly displays only two Raman-active modes: the $\mathrm{E}_{\mathrm{g}}$ mode, involving the periodic, antiparalell motion of oxygen atoms within adjacent O-layers, and a higher wavenumber $A_{1 g}$ mode where oxygen atoms move along the lattice's c-axis. ${ }^{32,33}$ Our DFT phonon calculations and experimental spectrum in Figure 2 indeed agree with the symmetry-based predictions: the $\mathrm{E}_{\mathrm{g}}$ and $\mathrm{A}_{1 \mathrm{~g}}$ bands are experimentally observed at $487 \mathrm{~cm}^{-1} 596 \mathrm{~cm}^{-1}$, respectively. 
a. GGA

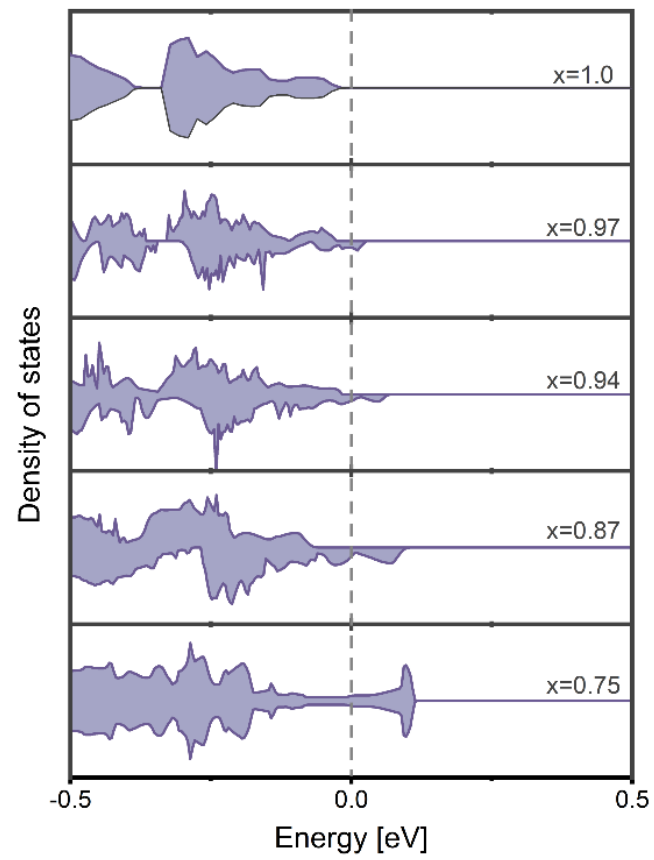

b. $G G A+U$

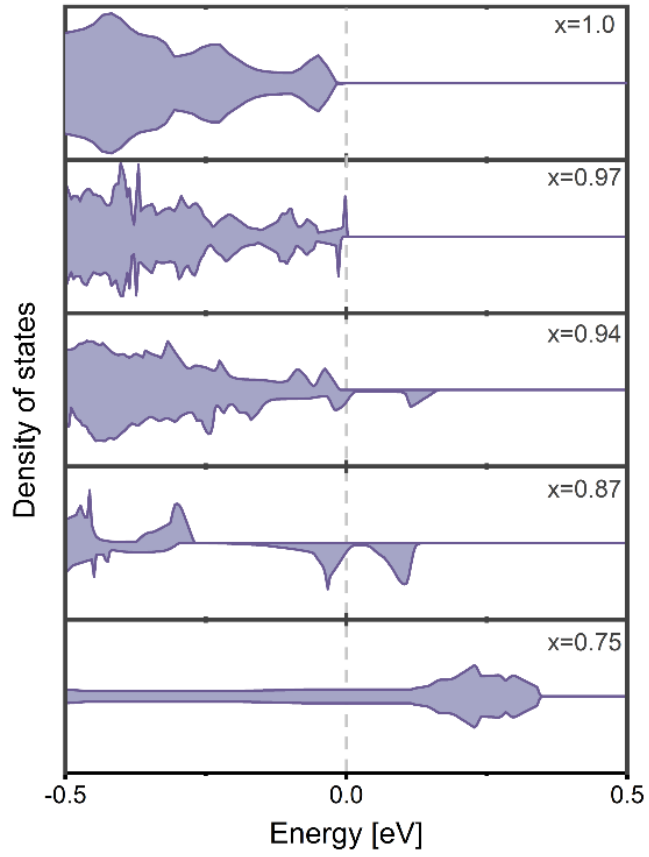

Figure 1. DFT-calculated total density of states of various $\mathrm{Li}_{x} \mathrm{CoO}_{2}$ supercells, using $\boldsymbol{a}$. GGA and $\boldsymbol{b}$. GGA+U functionals. The energy scale is zeroed vs. the Fermi Energy (dashed vertical line).

Notably, the $\mathrm{E}_{\mathrm{g}} / \mathrm{A}_{1 \mathrm{~g}}$ intensity ratio differs when changing the laser excitation wavelength (Figure 2). As the two lasers incide the sample with comparable power, the walenength dependency hints at Raman resonance effects. A Raman spectrum might be resonance-enhanced when the energy of the incident radiation coincides with the energy required to promote an electronic transition.

In agreement with most literature studies, we observe the $E_{g} / A_{1 g}$ intensity ratio to change from $\mathrm{E}_{\mathrm{g}} / \mathrm{A}_{1 \mathrm{~g}}>1$ under a $632 \mathrm{~nm}$ excitation, ${ }^{14,34,35}$ to $E_{g} / A_{1 g}<1$ under $514 \mathrm{~nm}^{36-41}, 532 \mathrm{~nm}^{14,42-44}$ and even $785 \mathrm{~nm}^{45}$ excitations. Note that our DFT-calculated Raman activities exhibit the same $\mathrm{E}_{\mathrm{g}} / \mathrm{A}_{1 \mathrm{~g}}<1$ intensity ratio as the experimental spectrum under $532 \mathrm{~nm}$ excitation (Figure 2). Since phonon calculations are agnostic to electronic excitations, this implies that the $\mathrm{E}_{\mathrm{g}} / \mathrm{A}_{1 \mathrm{~g}}<1$ intensity ratio represents non-resonant conditions. Therefore, we conclude that the Raman spectrum of fully lithiated LCO is resonance-enhanced under $632 \mathrm{~nm}$

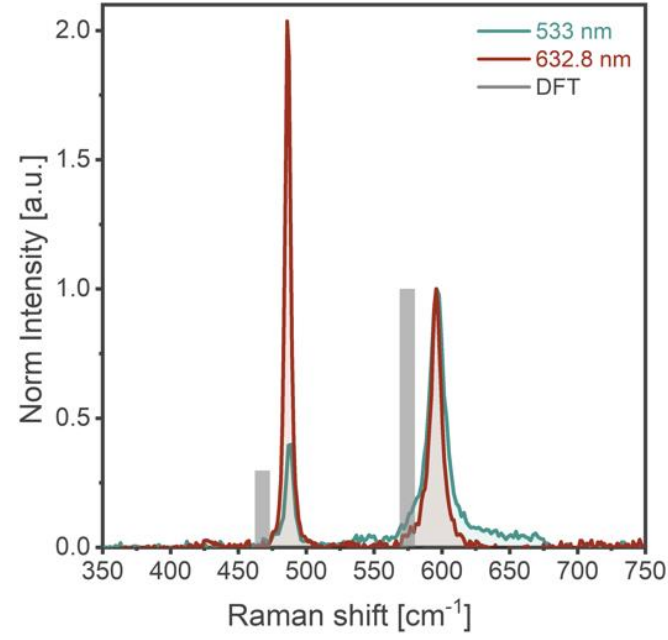

Figure 2. Raman spectra of fully lithiated $\mathrm{LiCoO}_{2}$ under different excitation energies and predicted by DFT phonon calculations. Each spectrum is normalized to the intensity of its $E_{g}$ band.

excitation. This is in contrast to the study of Gross et al., who suggested Raman enhancement under $532 \mathrm{~nm}$ wavelength, based on their observation of overtone bands,${ }^{14}$ which are typical features of resonance effects. ${ }^{46}$ Our systematic investigation of the experimental and DFT-calculated absorption spectra of $\mathrm{LiCoO}_{2}$ (Supp. Fig. 3) suggests that both laser excitations can cause Raman resonance. Further elucidation of this wavelengthdependency requires i) in the theory side, an explicit accounting of vibronic interactions and ii) in the experimental side, studying the $\mathrm{E}_{\mathrm{g}} / \mathrm{A}_{1 \mathrm{~g}}$ bands and overtones under more excitation wavelengths - ideally using single crystals. ${ }^{15}$

In the next section, we monitor how delithiation influences the appearance of the spectra under 633 and $532 \mathrm{~nm}$ excitations.

\section{Raman spectra of $\mathrm{Li}_{x} \mathrm{CoO}_{2}$ during the phase transition}

Figure 3a summarizes the operando Raman experiment conducted with the $633 \mathrm{~nm}$ laser excitation. The contour map representing the operando Raman spectra clearly shows both $\mathrm{E}_{\mathrm{g}}$ and $\mathrm{A}_{1 \mathrm{~g}}$ bands disappearing early in the delithiation process, and no new bands being distinguished thereafter. The fitted peak positions remain fairly constant until the bands disappear; the scattered datapoints are algorithm attempts to fit noise. The fitted peak intensities show a weakening trend from the very beginning of delithiation until completely disappearing when the LCO lithium content $x$ falls below 0.93 . Figure 3b shows the same intensity fading under $532 \mathrm{~nm}$ laser excitation, with the key difference that the $\mathrm{E}_{\mathrm{g}} / \mathrm{A}_{1 \mathrm{~g}}$ bands do not disappear completely after the phase transition.

Many authors argued that the drastic decrease in Raman intensities upon delithiation stems from the metallic behavior of the $\beta$-phase $\mathrm{Li}_{0.75} \mathrm{CoO}_{2} \cdot{ }^{36,47-50}$ In general, when light incides onto a conductive material, most of it is scattered and the small fraction that does enter the sample, dampens quickly. By modelling light penetration based on classical electrodynamics (see supplementary Information: light attenuation model), we indeed find that the skin depth of the conductive $\beta$-phase becomes shallower than the calculated confocal depth of focus determined by the microscope optics ${ }^{16}$ (c.a. $4.2 \mu \mathrm{m}$ under both laser excitations). Therefore, the Raman signal penetration in the metallic material is shallower than the confocal depth of focus. However, the Raman intensities under $633 \mathrm{~nm}$ excitation disappear completely, while under $533 \mathrm{~nm}$ excitation the bands are still detected, even though both excitations are expected to penetrate into the sample to the same depth. Therefore, the skin effect is not the only physical mechanism causing the decrease in intensities. 

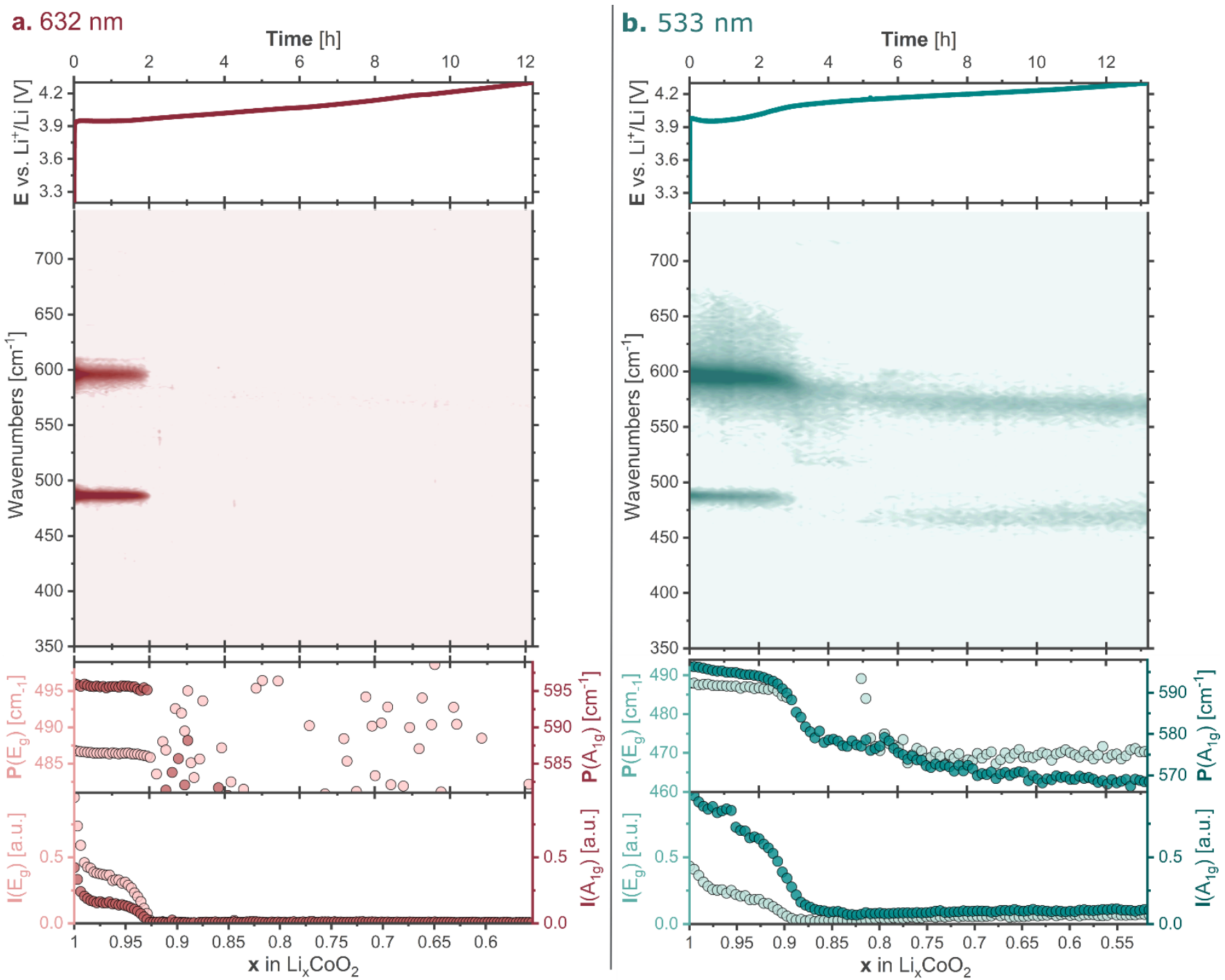

Figure 3. Constant current profiles (top), contour maps of the operando Raman spectra (middle), fitted peak positions and intensities (bottom) of $\mathrm{Li}_{x} \mathrm{CoO}_{2}$ during the first delithiation $\left(10 \mathrm{~mA} / \mathrm{g}\right.$, $3.0 \mathrm{to} 4.3 \mathrm{Vvs}$. $\left.\mathrm{Li}^{+} / \mathrm{Li}\right)$ as recorded using $\mathrm{a} .633 \mathrm{~nm}$ laser excitation and $\mathrm{b} .533 \mathrm{~nm}$ laser excitation.

In summary, both the $\mathrm{E}_{\mathrm{g}}$ and $\mathrm{A}_{1 \mathrm{~g}}$ bands are still detectable for most of the delithiation process under $533 \mathrm{~nm}$ excitation, but not under $633 \mathrm{~nm}$ excitation. Our results agree with similar studies that report absent bands when probing delithiated LCO with a $633 \mathrm{~nm}$ laser, ${ }^{51}$ but detectable $\mathrm{E}_{\mathrm{g}}$ and $\mathrm{A}_{1 \mathrm{~g}}$ bands when probed with $514 \mathrm{~nm}^{36,39,41}$ or $533 \mathrm{~nm}^{43,44,52}$ excitations. Therefore, the Raman intensities could be resonance-enhanced by the $532 \mathrm{~nm}$ excitation when LCO is partially delithiated, but enhanced by the $633 \mathrm{~nm}$ excitation instead when LCO is fully lithiated.

Given the drastic electronic changes associated to the insulatormetal transition, it is plausible that the resonance frequency of LCO changes during delithiation as well: from c.a. $633 \mathrm{~nm}$ at $\mathrm{SOL}=1.00$ to c.a. $532 \mathrm{~nm}$ at $\mathrm{SOL}<0.75$. Indeed, our computed absorption spectra (Fig. S4) show additional peaks emerging below $1 \mathrm{eV}$ upon delithiation, which could enable new vibronic transitions that remain forbidden when LCO is fully lithiated. The rich wavelength-dependent features observed in Figure 3 could open the door for a more comprehensive exploration of the electronic structure of LCO upon cycling.

\section{Raman spectra of $\mathrm{Li}_{\mathrm{x}} \mathrm{CoO}_{2}$ after the phase transition}

After the phase transition completes at $\mathrm{SOL}=0.75$ (Figure $3 \mathrm{~b}$ ), both $\mathrm{E}_{\mathrm{g}}$ and $\mathrm{A}_{1 \mathrm{~g}}$ bands appear at ca. 470 and $575 \mathrm{~cm}^{-1}$, respectively; i.e., red-shifted ca. $20 \mathrm{~cm}^{-1}$ from their positions in fully lithiated LCO. Shifts on the $\mathrm{E}_{\mathrm{g}} / \mathrm{A}_{1 \mathrm{~g}}$ modes of layered materials are associated to the strengthening (blue-shifts) or weakening (red-shifts) of the interatomic forces pinning oxygen atoms to their equilibrium positions within the lattice. ${ }^{53}$ Therefore, oxygen atoms experience a bond weakening during the phase transition, possibly because the $\mathrm{Li}^{+}$depletion weakens the electrostatic attraction between $\mathrm{Li}^{+}$and $\mathrm{O}^{2-}$. Such bond weakening might also result in expanded lattice parameters of Li-deficient LCO, as indeed shown by X-ray diffraction experiments. ${ }^{9}$

As delithiation proceeds below $\mathrm{SOL}<0.75$, the $\mathrm{E}_{\mathrm{g}}$ band position remains constant; instead, the $\mathrm{A}_{1 \mathrm{~g}}$ band shifts slightly but steadily towards lower wavenumbers (Figure $3 b$ ). These observations suggests that the interatomic forces keep weakening, but this time only along the c-axis (the $\mathrm{A}_{1 \mathrm{~g}}$ displacement vectors align with the lattice's c-axis, see Supplementary Figure S2). In addition, the intensities of both bands show a slight increasing trend that suggests an increase in electron density around oxygen atoms ${ }^{13,53}$, consistent with the slightly increased Co-O covalency observed from X-ray absorption spectra. ${ }^{54}$ In summary, the position and intensities of the $A_{1 g}$ and $E_{g}$ bands suggest that delithiation between $0.75>\mathrm{SOL}>0.50$ weakens oxygen interactions while increasing its electron density, which precedes the observed oxygen loss from the structure $\mathrm{SOL}<0.5$. $^{55}$

\section{Dynamics of the phase transition}

During the phase transition, $\alpha$-LCO (i.e. $\mathrm{Li}_{0.93} \mathrm{CoO}_{2}$ ) generally features intense $E_{g}$ and $A_{1 g}$ Raman bands while $\beta$-LCO (i.e. $\mathrm{Li}_{0.75} \mathrm{CoO}_{2}$ ) has weak (or undetectable) bands. Hence, independently of the cause, the changes in intensity can be used 


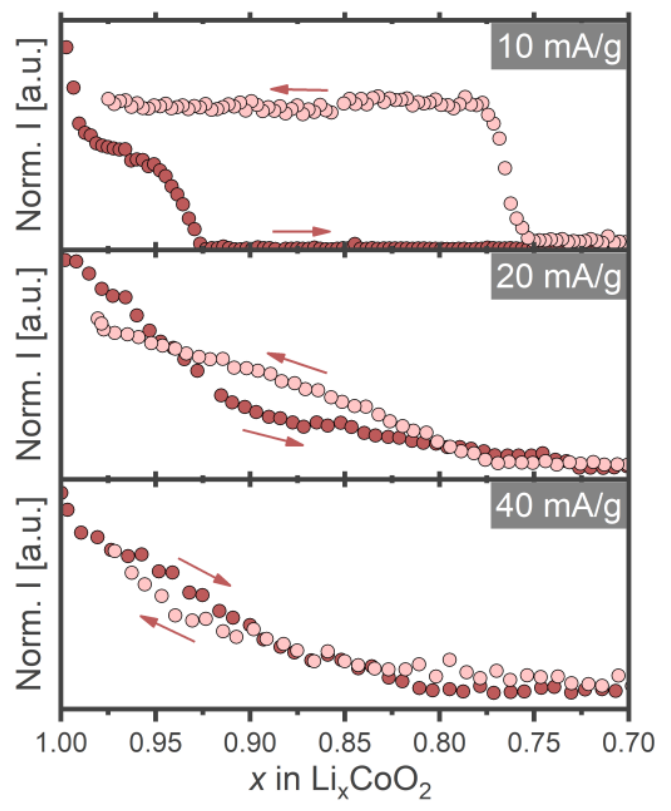

Figure 4. Fitted peak intensities of $\mathrm{Li}_{x} \mathrm{CoO}_{2} \mathrm{E}_{\mathrm{g}}$ Raman-active band during the first cycle at 10,20 and $40 \mathrm{~mA} / \mathrm{g}$. Each data point correspond to the fitted intensity from a single spectrum.

to monitor the evolving proportion of $\alpha$ and $\beta$ phases. Figure 3 shows that during delithiation the intensities drop following an S-shaped trend rather than monotonically. The process responsible for the trends appears unrelated to the electronic nature of the phase transition, because the same trend is observed under both excitation wavelengths. We therefore conducted additional operando experiments to further investigate the origin of these phenomena.

Figure 4 shows the Raman intensity of the $E_{g}$ band detected under $633 \mathrm{~nm}$ excitiation, at different cycling rates and during a full cycle. The most prominent feature is the hyteresis between delithiation and lithiation, which appears well-defined at 10 $\mathrm{mA} / \mathrm{g}$ but narrows at higher rates until virtually disappearing at $40 \mathrm{~mA} / \mathrm{g}$.

It is known that the electrochemical potential of CAMs feature hysteretic behaviors when de(lithiation) is not spatially homogeneous, either due to intra-particle concentration gradients or to first-order phase transitions. ${ }^{11,56}$ Likewise, a spatially-heterogeneous (de)lithiation within the CAM could cause hysteretic intensity trends by, for example, exposing a $\mathrm{Li}$ deficient particle shell (weak scatterer) to the probing laser, while simoultaneously reducing access to the Raman signals from a Li-rich (strong scatterer) core. Hysteretic trends could also reflect shifts between particle and electrode SOLs, originating from lateral and axial SOL heterogeneities at the electrode level. ${ }^{57,58} \mathrm{We}$ investigate these hypotheses by numerically modelling the laser's sampling volume -a proxy for the Raman intensities- and how it grows/shrinks upon incidence on a single particle of CAM with a spatially heterogeneous lithium concentration. The details of the model can be found in Supporting information: sampling volume model.

Figure 5 shows the simulated evolution of the sampling volume under three different phase transition scenarios. In a core-shell transition scenario (Figure 5a), the $\alpha$ and $\beta$ phases within a single particle of CAM are separated by a sharp boundary that propagates radially as the phase transition proceeds. The $\beta$-phase is assumed to enable limited light penetration without scattering Raman signals (supported by the observations in Figure 3a), so the resulting sampling volume (orange cone in Figure 5a) is that inciding upon the $\alpha$-phase. In this scenario, delthiation below $\mathrm{x}=0.93$ promotes the growth of a Li-deficient shell, which dampens the laser's access to the Li-rich (strong-scatterer) core, effectively reducing the sampling volume. As the phase
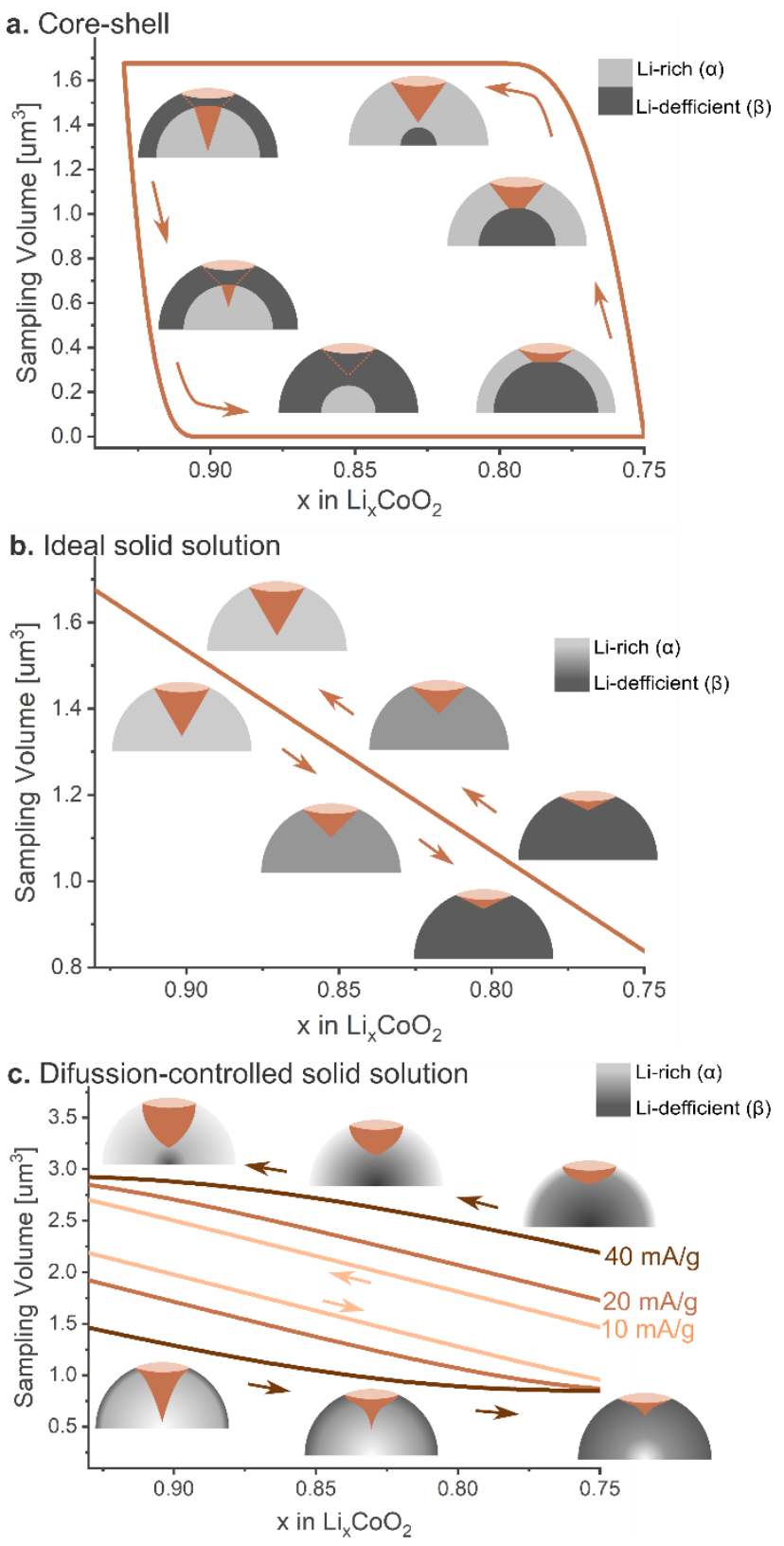

Figure 5. Simulated sampling volumes under three intra-particle heterogeneity scenarios: $\boldsymbol{a}$. core-shell spatial heterogeneity, $\boldsymbol{b}$. ideal solid solution with no spatial heterogeneity and $c$. diffusion-control Li gradients. Li-rich and Li-deficient zones within the simulated particles are represented in light and dark grey, respectively, while the orange cones represent the sampling volume.

boundary moves beyond the penetration depth of the $\beta$-phase, all access to the $\alpha$-phase core is lost and the sampling volume effectively vanishes. On relithiation the process reverses: a Lirich shell of $\alpha$-phase grows and with it the sampling volume, which reaches its maximum value when the Li-rich shell grows beyond the penetration depth in the $\alpha$-phase. The hysteresis emerges because the sampling volume vanishes at ca. $\mathrm{x}=0.9$, before delithiation completes the phase transition at $\mathrm{x}=0.75$; likewise, the sampling volume reaches its maximum value before relithiation completes the transition at $\mathrm{x}=0.93$.

The transition might also proceed as an ideal solid solution (Figure 5b), where the $\alpha$ and $\beta$ phases are completely miscible. The particle would exhibit a spatially homogeneous SOL changing linearly during (de)lithiation. In this scenario, the sampling volume also changes linearly without any hystheresis. The third simulation (Figure 5c) accounts for the scenario where the $\alpha$ and $\beta$ phases are miscible but diffusion limitations cause intra-particle Li gradients. Just as in the first scenario, the laser penetrates into a spatially-heterogeneous environment; hence, 
the sampling volume reaches its maximum and minium values before the transition is completed, resulting in hysteretic trends. Moreover, our Fick's diffusion-based approach to model Ligradients enables us to simulate the effects of multiple cycling rates. When the Li-gradients expectedly become more pronounced at higher rates, the sampling volume hystheresis widens as the rate accelerates from 10 to $40 \mathrm{~mA} / \mathrm{g}$ (see Figure 5c).

\section{Phase transition pathways: core-shell vs. solid solution}

We find two key conclusions by comparing the three simulated scenarios to our experimental results. First, while the spatial heterogeneities predict sampling volume hysthereses (Figure $5 \mathrm{a}, \mathrm{c})$, intra-particle Li-gradients ${ }^{59}$ and inter-particle SOL heterogeneities ${ }^{60}$ would widen the hysthereses at higher rates, not narrow them as experimentally observed in Figure 4. Based on this contradiction, we discard intra- and inter-particle heterogeneites and thus suggest that the intensity hystheresis observed at $10 \mathrm{~mA} / \mathrm{g}$ rather originates from a core-shell phase separation. We emphasize that, while diffraction experiments have crystallographically distinguished the $\alpha$ and $\beta$ phases, ${ }^{9}$ the depth-resolved nature of our Raman experiments further enables elucidating that the core-shell geometry occurs within a single CAM particle. In support, Zhang and White demonstrated that the constant-current curves of a porous LCO electrode are better modelled assuming that the phase transition proceeds via a coreshell mechanism with a sharp phase boundary. ${ }^{61}$

The second key conclusion is related to the rate-dependent changes of the intensity trends in Figure 4: the negligible intensity hystheresis experimentally observed at $40 \mathrm{~mA} / \mathrm{g}$ resembles the one modelled for a solid solution phase transition (Figure 5b). Therefore, our observations provide compelling evidence that the near-equilibrium $(10 \mathrm{~mA} / \mathrm{g}$ ) core-shell (twophase) route of the insulator-metal transition is suppressed in favor of a solid-solution route at higher cycling rates $(40 \mathrm{~mA} / \mathrm{g}$, approx. C/5). Rate-dependent in situ diffraction studies on $\mathrm{LiCoO}_{2}$ indeed show clearly separated (003) Bragg reflections from the $\alpha$ and $\beta$ phases at low cycling rates $(<C / 10)$, which generally appear merged as a single (003) reflection at higher cycling rates $(>\mathrm{C} / 10) .{ }^{62,63}$

Rate-dependent pathways of phase transitions are not unprecedented. For instance, both $\mathrm{LiNi}_{0.33} \mathrm{Co}_{0.33} \mathrm{Mn}_{0.33} \mathrm{O}_{2}$ and $\mathrm{LiFePO}_{4} \mathrm{CAMs}$ cycle following thermodynamically-favoured two-phase transition pathways at low rates, which change to a non-equilibrium, solid-solution transition pathway at higher rates, assisted by the associated overpotentials. ${ }^{64,65}$ Our $\mu \mathrm{m}$ resolved results indicate that for $\mathrm{LiCoO}_{2}$ such pathway change occurs within single particles of CAM at rates as low as C/10. Furthermore, observing a kinetically-stabilized solid-solution CAM composition is only possible through operando measurements: stopping the cycle for in situ/ex situ measuring would cause the system to separate into the $\alpha$ - and $\beta$-phases due to thermodynamic relaxation.

The suppression of the two-phase pathway in favour of a solidsolution route potentially explains why LCO exhibits competitive rate capabilities. The CAM is thermodynamically prone to cycle via a kinetically slow two-phase transition, but as the cycling current increases to practical rates, the transition follows a faster, solid-solution route.

\section{Conclusions}

Herein, the insulator-metal transition of LCO is explored by operando Raman spectroscopy, complemented with DFT calculations and a newly-developed sampling volume model. Based on our GGA $(+U)$-DFT calculations, we confirm that LCO behaves as a Mott insulator at dilute Li-vacancy concentrations $(x>0.87)$ and then transforms into a metallic phase at $x<0.75$. The wavelength-dependent Raman spectra of $\mathrm{Li}_{\mathrm{x}} \mathrm{CoO}_{2}$ are found to be enhanced by electronic resonance and are thus sensitive to the non-rigid behavior of the material's electronic structure during cycling. We record hundreds of operando Raman spectra of $\mathrm{LCO}$ cycled at $10 \mathrm{~mA} / \mathrm{g}(\sim \mathrm{C} / 20)$, and find the fitted peak intensities to outline hysteretic trends during the insulator-metal phase transition. By repeating the operando Raman measurements at higher rates we show that the hystheresis, surprisingly, narrows at higher cycling rates. We developed a simple numerical model to investigate the effect that multiple $\mathrm{Li}^{+}$ heterogeneities would have on the sampling volume - a proxy for Raman intensities. By comparing the model results to the experimentally observed trends, we provide compelling evidence that the insulator-metal transition of $\mathrm{LiCoO}_{2}$ follows a two-phase route at $10 \mathrm{~mA} / \mathrm{g}(\sim \mathrm{C} / 20)$, which is suppressed in favor of a solid-solution route at rates above $20 \mathrm{~mA} / \mathrm{g}(\sim \mathrm{C} / 10)$. Our reslts indicate that LCO exhibits competitive rate capabilities because the material gains access to a kineticallyfaster solid-solution transition route as it is cycled at practical rates. We anticipate the solid solution route to be only observable through operando measurements, since without a current bias the CAM particle would relax to its thermodynamically-favoured two-phase state; we thus emphasize the essential role that operando techniques play in unravelling the dynamic processes of Li-ion active materials.

\section{Associated Content}

- Supporting information: electrochemical profiles, Raman-active vibrations, DFT-calculated and UV-Vis absorption spectra.

- Supporting information: Penetration depth model.

- Supporting information: Sampling volume model.

\section{Author Information}

*eibfl@dtu.dk

Notes

- The authors declare no competing financial interests.

- EF current address: Department of Energy Conversion and Storage, Technical University of Denmark (DTU), Anker Engelunds Vej, 2800 Kgs. Lyngby, Denmark.

- NM current address: Department of Physics, Chalmers University of Technology, SE 412 96, Göteborg, Sweden.

\section{References}

1. Lu, L., Han, X., Li, J., Hua, J. \& Ouyang, M. A review on the key issues for lithium-ion battery management in electric vehicles. $J$. Power Sources 226, 272-288 (2013).

2. Amatucci, G. G., Tarascon, J. M. \& Klein, L. C. Cobalt dissolution in LiCoO2-based non-aqueous rechargeable batteries. Solid State Ionics 83, 167-173 (1996).

3. Ensling, D. et al. Nonrigid band behavior of the electronic structure of $\mathrm{LiCoO} 2$ thin film during electrochemical Li deintercalation. Chem. Mater. 26, 3948-3956 (2014).

4. Kannan, A. M., Rabenberg, L. \& Manthiram, A. High capacity surface-modified $\mathrm{LiCoO} 2$ cathodes for lithium-ion batteries. Electrochem. Solid-State Lett. 6, 16-19 (2003).

5. Liu, Q. et al. Approaching the capacity limit of lithium cobalt oxide in lithium ion batteries via lanthanum and aluminium doping. Nat. Energy 3, 936-943 (2018).

6. Lyu, Y. et al. An Overview on the Advances of LiCoO2 Cathodes for Lithium-Ion Batteries. Adv. Energy Mater. 11, 1-29 (2021).

7. Fergus, J. W. Recent developments in cathode materials for lithium ion batteries. J. Power Sources 195, 939-954 (2010).

8. Dokko, K., Nakata, N. \& Kanamura, K. High rate discharge capability of single particle electrode of LiCoO2. J. Power Sources 189, 783-785 (2009) 

Diffraction Studies of Lithium Intercalation in $\mathrm{Li}_{x} \mathrm{CoO}_{2} . J$. Electrochem. Soc. 139, 2091 (1992).

10. Van der Ven, A., Bhattacharya, J. \& Belak, A. A. Understanding Li Diffusion in Li-Intercalation Compounds. Acc. Chem. Res. 46, 1216-1225 (2013).

11. Radin, M. D. et al. Narrowing the Gap between Theoretical and Practical Capacities in Li-Ion Layered Oxide Cathode Materials. Adv. Energy Mater. 7, 1-33 (2017).

12. Flores, E., Vonrüti, N., Novák, P., Aschauer, U. \& Berg, E. J. Elucidation of $\mathrm{Li}_{\mathrm{x}} \mathrm{Ni}_{0.8} \mathrm{Co}_{0.15} \mathrm{Al}_{0.05} \mathrm{O}_{2}$ Redox Chemistry by Operando Raman Spectroscopy. Chem. Mater. 30, 4694-4703 (2018).

13. Flores, E., Novák, P., Aschauer, U. \& Berg, E. J. Cation Ordering and Redox Chemistry of Layered Ni-Rich Li x Ni 1-2 y Co y Mn y O 2: An Operando Raman Spectroscopy Study. Chem. Mater. 32, 186-194 (2020).

14. Gross, T. \& Hess, C. Raman diagnostics of LiCoO2 electrodes for lithium-ion batteries. J. Power Sources 256, 220-225 (2014).

15. Flores, E., Novák, P. \& Berg, E. J. In situ and Operando Raman spectroscopy of layered transition metal oxides for Li-ion battery cathodes. Front. Energy Res. 6, 1-16 (2018).

16. Everall, N. J. Confocal Raman microscopy: Performance, pitfalls, and best practice. Appl. Spectrosc. 63, (2009).

17. Boulet-Roblin, L. et al. Versatile approach combining theoretical and experimental aspects of raman spectroscopy to investigate battery materials: the case of the $\mathrm{LiNi}_{0.5} \mathrm{Mn}_{1.5} \mathrm{O}_{4}$ spinel. J. Phys. Chem. C 120, 16377-16382 (2016).

18. Kresse, G. \& Hafner, J. Ab initio molecular-dynamics simulation of the liquid-metal-amorphous-semiconductor transition in germanium. Phys. Rev. B 49, 14251-14269 (1994).

19. Kresse, G. \& Furthmüller, J. Efficiency of ab-initio total energy calculations for metals and semiconductors using a plane-wave basis set. Comput. Mater. Sci. 6, 15-50 (1996).

20. Kresse, G. \& Hafner, J. Ab initio molecular dynamics for liquid metals. Phys. Rev. B 47, 558-561 (1993).

21. Perdew, J. P., Burke, K. \& Ernzerhof, M. Generalized gradient approximation made simple. Phys. Rev. Lett. 77, 3865-3868 (1996).

22. Blöchl, P. E. Projector augmented-wave method. Phys. Rev. B 50, 17953-17979 (1994).

23. Joubert, D. From ultrasoft pseudopotentials to the projector augmented-wave method. Phys. Rev. B - Condens. Matter Mater. Phys. 59, 1758-1775 (1999).

24. Anisimov, V. I., Zaanen, J. \& Andersen, O. K. Band theory and Mott insulators: Hubbard U instead of Stoner I. Phys. Rev. B 44, 943-954 (1991).

25. Dudarev, S. L., Botton, G. A., Savrasov, S. Y., Humphreys, C. J. \& Sutton, A. P. Electron-energy-loss spectra and the structural stability of nickel oxide: An LSDA+U study. Phys. Rev. B 57, 1505-1509 (1998).

26. Hoang, K. \& Johannes, M. Defect Physics and Chemistry in Layered Mixed Transition Metal Oxide Cathode Materials: (Ni,Co,Mn) vs (Ni,Co,Al). Chem. Mater. 28, 1325-1334 (2016).

27. Togo, A., Oba, F. \& Tanaka, I. First-principles calculations of the ferroelastic transition between rutile-type and $\mathrm{CaCl} 2$-type $\mathrm{SiO} 2$ at high pressures. Phys. Rev. B - Condens. Matter Mater. Phys. 78, 1-9 (2008).

28. Fonari, A. \& Stauffer, S. vasp_raman.py. (2013).

29. Ménétrier, M., Saadoune, I., Levasseur, S. \& Delmas, C. The insulator-metal transition upon lithium deintercalation from $\mathrm{LiCoO}_{2}$ : electronic properties and ${ }^{7} \mathrm{Li}$ NMR study. J. Mater. Chem. 9, 1135-1140 (1999).

30. Imanishi, N., Fujiyoshi, M., Takeda, Y., Yamamoto, O. \& Tabuchi, M. Preparation and 7Li-NMR study of chemically delithiated Li1-xCoO2 (0. Solid State Ionics 118, 121-128 (1999).

31. Marianetti, C. A., Kotliar, G. \& Ceder, G. A first-order Mott transition in LixCoO2. Nat. Mater. 3, 627-631 (2004).

32. Flores, E. Development of operando diagnostics for Li-ion cathodes by Raman spectroscopy. (2019) doi:10.3929/ethz-b000373382 .

33. Julien, C. Local cationic environment in lithium nickel-cobalt oxides used as cathode materials for lithium batteries. Solid State Ionics 136-137, 887-896 (2000).
34. Okubo, M. et al. Nanosize effect on high-rate Li-ion intercalation in LiCoO2 electrode. J. Am. Chem. Soc. 129, 7444-7452 (2007).

35. Vandenberg, A. \& Hintennach, A. A comparative microwaveassisted synthesis of carbon-coated $\mathrm{LiCoO} 2$ and $\mathrm{LiNiO} 2$ for lithium-ion batteries. Russ. J. Electrochem. 51, 310-317 (2015).

36. Itoh, T., Sato, H., Nishina, T., Matue, T. \& Uchida, I. In situ Raman spectroscopic study of $\mathrm{Li}_{\mathrm{x}} \mathrm{CoO}_{2}$ electrodes in propylene carbonate solvent systems. J. Power Sources 68, 333-337 (1997).

37. Zhang, Y., Lu, Z. G., Chung, C. Y. \& Zhu, M. Kinetics of Li+transport and capacity retention capability of HTLiCoO2films. Phys. Scr. T T129, 38-42 (2007).

38. Kushida, K. \& Kuriyama, K. Optical absorption related to Co-3d bands in sol-gel grown LiCoO2 films. Solid State Commun. 118 615-618 (2001).

39. Matsuda, Y. et al. In situ Raman spectroscopy of LixCoO2 cathode in $\mathrm{Li} / \mathrm{Li} 3 \mathrm{PO} 4 / \mathrm{LiCoO} 2$ all-solid-state thin-film lithium battery. Solid State Ionics 335, 7-14 (2019).

40. Song, S., Han, K., Fujita, H. \& Yoshimura, M. In situ visible Raman spectroscopic study of phase change in $\mathrm{LiCoO} 2$ film by laser irradiation. Chem. Phys. Lett. 344, 299-304 (2001).

41. Inaba, M., Iriyama, Y., Ogumi, Z., Todzuka, Y. \& Tasaka, A. Raman study of layered rock-salt LiCoO2 and its electrochemical lithium deintercalation. J. Raman Spectrosc. 28, 613-617 (1997).

42. Mendoza, L., Baddour-Hadjean, R., Cassir, M. \& Pereira-Ramos, J. P. Raman evidence of the formation of LT-LiCoO2 thin layers on $\mathrm{NiO}$ in molten carbonate at $650^{\circ} \mathrm{C}$. Appl. Surf. Sci. 225, $356-$ 361 (2004).

43. Fukumitsu, H., Omori, M., Terada, K. \& Suehiro, S. Development of in situ cross-sectional Raman imaging of $\mathrm{LiCoO} 2$ cathode for Li-ion battery. Electrochemistry 83, 993-996 (2015).

44. Liu, H. L. et al. Electronic structure and lattice dynamics of $\mathrm{Li}_{\mathrm{x}} \mathrm{CoO}_{2}$ single crystals. New J. Phys. 17, 103004 (2015).

45. Le Van-Jodin, L. et al. Ex situ and operando study of $\mathrm{LiCoO} 2$ thin films by Raman spectroscopy: Thermal and electrochemical properties. J. Raman Spectrosc. 50, 1594-1601 (2019).

46. Clark, R. J. H. \& Dines, T. J. Resonance Raman Spectroscopy, and Its Application to Inorganic Chemistry. New Analytical Methods (27). Angew. Chemie Int. Ed. English 25, 131-158 (1986).

47. Inaba, M., Iriyama, Y., Ogumi, Z., Todzuka, Y. \& Tasaka, A. Raman study of layered rock-salt $\mathrm{LiCoO} 2$ and its electrochemical lithium deintercalation. J. Raman Spectrosc. 28, 613-617 (1997).

48. Itoh, T. et al. Spectroelectrochemical studies on highly polarized LiCoO2 electrode in organic solutions. Electrochem. commun. 2, 743-748 (2000).

49. Baddour-Hadjean, R. \& Pereira-Ramos, J. P. Raman Investigation of Cathode Materials for Lithium Batteries. Lithium Ion Rechargeable Batteries: Materials, Technology, and New Applications (2010). doi:10.1002/9783527629022.ch6.

50. Novák, P. et al. Advanced in situ methods for the characterization of practical electrodes in lithium-ion batteries. J. Power Sources 90, 52-58 (2000).

51. Zhou, Y. et al. Observation of Interfacial Degradation of Li6PS5Cl against Lithium Metal and $\mathrm{LiCoO} 2$ via In Situ Electrochemical Raman Microscopy. Batter. Supercaps 647-652 (2020) doi:10.1002/batt.201900218.

52. Otoyama, M., Ito, Y., Hayashi, A. \& Tatsumisago, M. Raman imaging for $\mathrm{LiCoO}_{2}$ composite positive electrodes in all-solid-state lithium batteries using $\mathrm{Li}_{2} \mathrm{~S}-\mathrm{P}_{2} \mathrm{~S}_{5}$ solid electrolytes. J. Power Sources 302, 419-425 (2016).

53. Flores, E., Vonrüti, N., Novák, P., Aschauer, U. \& Berg, E. J. Elucidation of LixNi0.8Co0.15Al0.05O2 Redox Chemistry by Operando Raman Spectroscopy. Chem. Mater. 30, 4694-4703 (2018).

54. Yoon, W. S. et al. Oxygen contribution on Li-ion intercalationdeintercalation in $\mathrm{LiCoO} 2$ investigated by $\mathrm{O} \mathrm{K}$-edge and $\mathrm{Co} \mathrm{L}$ edge X-ray absorption spectroscopy. J. Phys. Chem. B 106, 25262532 (2002).

55. Lundström, R. \& Berg, E. J. Design and validation of an online partial and total pressure measurement system for Li-ion cells. $J$. Power Sources 485, 229347 (2021).

56. Dreyer, W. et al. The thermodynamic origin of hysteresis in insertion batteries. Nat. Mater. 9, 448-453 (2010).

57. Nishi, T., Nakai, H. \& Kita, A. Visualization of the State-ofCharge Distribution in a LiCoO 2 Cathode by In Situ Raman 
Imaging. J. Electrochem. Soc. 160, A1785-A1788 (2013).

58. Sasaki, T., Villevieille, C., Takeuchi, Y. \& Novák, P. Understanding Inhomogeneous Reactions in Li-Ion Batteries: Operando Synchrotron X-Ray Diffraction on Two-Layer Electrodes. Adv. Sci. 2, 2-7 (2015).

59. $\mathrm{Xu}, \mathrm{Y}$. et al. In situ Visualization of State-of-Charge Heterogeneity within a LiCoO2 Particle that Evolves upon Cycling at Different Rates. ACS Energy Lett. 2, 1240-1245 (2017).

60. Liu, J., Kunz, M., Chen, K., Tamura, N. \& Richardson, T. J. Visualization of charge distribution in a lithium battery electrode. J. Phys. Chem. Lett. 1, 2120-2123 (2010).

61. Zhang, Q. \& White, R. E. Moving Boundary Model for the Discharge of a LiCoO[sub 2] Electrode. J. Electrochem. Soc. 154, A587 (2007).

62. Liu, L. et al. Electrochemical and In Situ Synchrotron XRD Studies on $\mathrm{Al}[$ sub 2] $\mathrm{O}$ [sub 3]-Coated $\mathrm{LiCoO}[$ sub 2] Cathode Material. J. Electrochem. Soc. 151, A1344 (2004).

63. Chung, K. Y. et al. Structural Studies on the Effects of $\mathrm{ZrO}$ [sub 2] Coating on LiCoO[sub 2] during Cycling Using In Situ X-Ray Diffraction Technique. J. Electrochem. Soc. 153, A2152 (2006).

64. Liu, H. et al. Capturing metastable structures during high-rate cycling of LiFePO 4 nanoparticle electrodes. Science (80-. ). 344, (2014).

65. Zhou, Y. N. et al. High-Rate Charging Induced Intermediate Phases and Structural Changes of Layer-Structured Cathode for Lithium-Ion Batteries. Adv. Energy Mater. 6, 1-8 (2016). 